OPEN ACCESS

Edited by:

Kevin T. Ong,

Armadale Health Service, Australia

Reviewed by:

Masafumi Ihara,

National Cerebral and Cardiovascular

Center, Japan

Michael Woodward

University of Melbourne, Australia

*Correspondence:

Antoine M. Hakim

AHAKIM@toh.ca

Received: 12 March 2021 Accepted: 31 March 2021

Published: 29 April 2021

Citation:

Hakim AM (2021) A Proposed

Hypothesis on Dementia:

Inflammation, Small Vessel Disease,

and Hypoperfusion Is the Sequence

That Links All Harmful Lifestyles

to Cognitive Impairment.

Front. Aging Neurosci. 13:679837.

doi: 10.3389/fnagi.2021.679837

\section{A Proposed Hypothesis on Dementia: Inflammation, Small Vessel Disease, and Hypoperfusion Is the Sequence That Links All Harmful Lifestyles to Cognitive Impairment}

\author{
Antoine M. Hakim ${ }^{1,2 *}$ \\ 'Brain and Mind Research Institute, University of Ottawa, Ottawa, ON, Canada, ${ }^{2}$ Division of Neurology, University of Ottawa, \\ Ottawa, ON, Canada
}

There is growing consensus that certain lifestyles can contribute to cognitive impairment and dementia, but the physiological steps that link a harmful lifestyle to its negative impact are not always evident. It is also unclear whether all lifestyles that contribute to dementia do so through the same intermediary steps. This article will focus on three lifestyles known to be risk factors for dementia, namely obesity, sedentary behavior, and insufficient sleep, and offer a unifying hypothesis proposing that lifestyles that negatively impact cognition do so through the same sequence of events: inflammation, small vessel disease, decline in cerebral perfusion, and brain atrophy. The hypothesis will then be tested in a recently identified risk factor for dementia, namely hearing deficit. If further studies confirm this sequence of events leading to dementia, a significant change in our approach to this debilitating and costly condition may be necessary, possible, and beneficial.

Keywords: obesity, sedentary lifestyle, sleep insufficiency, inflammation, cerebral small vessel disease, cerebral blood flow, cognitive decline, a unifying hypothesis

\section{INTRODUCTION}

Dementia is a growing problem. It impacts the individual, his or her family, and the society they live in. Certain lifestyles have been confirmed to contribute to dementia, but the steps leading from the harmful lifestyle to its cognitive impact have not always been clear. This review proposes a stepwise progression from three selected lifestyles to their cognitive impact. It also suggests that the same process links all harmful lifestyles to their negative impacts on memory function.

Dementia represents a significant personal, family, and social burden, and the number of people with dementia is rising in many parts of the world (Patterson, 2018). One major factor leading to this increase is the longer life expectancy seen in many regions (Beltrán-Sánchez et al., 2015). As a result, the number of people with dementia has doubled since 1990 (GBD 2016; Dementia Collaborators, 2019). In contrast, there is growing consensus in the literature that dementia is not an inevitable companion to old age (Qiu and Fratiglioni, 2018). Rather, cognitive decline appears more likely when certain lifestyles have been present (Laurin et al., 2001; Serrano-Pozo and Growdon, 2019; Smith, 2019). This realization has shifted the scientific and social discourse in dementia from the search for a therapy to promoting prevention 
of the condition. The 2020 report of the Lancet Commission identified twelve potentially modifiable risk factors for dementia including less education, hypertension, hearing impairment, smoking, obesity, depression, physical inactivity, diabetes, and low social contact (Livingston et al., 2020), and suggested that $40 \%$ of worldwide dementias may be due to these factors.

Research has also provided two additional important observations relevant to the etiology of dementia. The first was that drugs that successfully eliminated cerebral accumulations of beta amyloid have so far shown only modest impact on cognitive deficits (Oxford et al., 2020), although trials are still ongoing. Ever since the original description that these proteins were present in the brains of individuals dying with dementia, they were considered to be etiologically significant in inducing dementia, and the modest impact they have had to date has forced a reappraisal of our approach to dementia.

The second landmark observation was that a decline in cerebral blood flow (CBF) was an early cerebral event that heralded the decline in cognitive function and may precede the appearance of the clinical syndrome by many years (IturriaMedina et al., 2016). This finding confirmed that vascular insufficiency is a major etiologic factor that anticipates the onset of cognitive deficits, and that the protein deposits found in the brain of demented individuals were more likely a consequence of the disease rather than its cause. While this was a major step forward in our understanding of the etiology of dementia, it left open the question: do all harmful lifestyles lead to cerebral hypoperfusion? If so, what are the physiological mechanisms that lead to the decline in CBF when a harmful lifestyle has persisted?

Several publications have proposed that inflammation may be the link between lifestyle, genetics, and Alzheimer's disease (Uzoni et al., 2015; Newcombe et al., 2018), but the mechanisms that link inflammation to this outcome are not clear. This review will focus on 3 lifestyle factors that negatively impact cognition, namely obesity, sedentary behavior, and insufficient sleep. In each case, a summary of the research associating the lifestyle to subsequent cognitive decline will be presented, and the impact of the lifestyle on cerebral vascular perfusion will be explored. The potential mechanisms linking the lifestyle to its eventual impact on perfusion will then be reviewed. A unifying hypothesis will be proposed, namely, that all lifestyles that negatively impact cognition do so through the activation of inflammatory factors, which then lead to small vessel disease, resulting in a reduction in cerebral perfusion and causing atrophy of structures essential for normal cognition (Figure 1). The evidence supporting this hypothesis for the three chosen lifestyles will be presented, and its potential application in the setting of hearing deficit, a newly identified risk factor for dementia, will then be explored. As well, the implications of this renewed understanding of dementia for the individual and for society will be presented.

\section{OBESITY}

After some debate on the matter, it is now well accepted that obesity is associated with cognitive decline (Whitmer et al., 2008).
The debate was triggered by a report that obesity may in fact reduce the risk for dementia (Qizilbash et al., 2015), but a number of methodological oversights were subsequently identified in this publication, pointing especially to the fact that a reduction in weight may occur in those affected by dementia (Singh-Manoux et al., 2018), implying that lower weight in later life may be due to the illness and consequently cannot be used to describe the association of obesity with dementia.

Studies recognizing that obesity in early and middle years negatively impact later cognitive abilities leave no doubt as to the long-term impact of obesity on memory functions, reestablishing obesity as a major risk factor for dementia (Xu et al., 2011; Kerti et al., 2013; Schwartz et al., 2013; Martin and Davidson, 2014).

The importance of this association is partly due to the growing problem of obesity in many jurisdictions. In the United States, in $2010,35.5 \%$ of men and $35.8 \%$ of women satisfied the criteria for obesity (body mass index (BMI) of 30 or more) (Flegal et al., 2012), with a clear trend for increasing prevalence of obesity between 1999 and 2012 in both men and women (Ford et al., 2014).

The presence of inflammatory mediators in obesity is so well established that adipose tissue is now considered to be an immune organ (Makki et al., 2013). In their extensive review on the topic of inflammation in obesity, Chait and Hartigh (2020) point out that inflammation associated with adipose tissue is chronic, sterile, low grade and impacts function of liver, muscle, and pancreas. Visceral adipose tissue is associated with secretion of inflammatory cytokines, and the elevated levels of C-reactive protein (CRP) and serum amyloid A are likely in response to the interleukin-6 (IL-6) secretion from the adipose tissue ( $\mathrm{Gu}$ et al., 2018b). As well, tumor necrosis factor alpha $(\mathrm{TNF} \alpha)$ levels positively correlate with adiposity, BMI, insulin levels, and insulin resistance (Zahorska-Markiewicz et al., 2000; Kern et al., 2001).

A positive feedback loop may link inflammation and obesity. Genetic polymorphisms near a central regulator of inflammatory cell function that coordinates inflammation is associated with obesity in humans, and silencing it in the laboratory setting reduces obesity (Karunakaran et al., 2020). This points to the complex relationship between inflammation and obesity.

Recent literature emphasizes the fact that small vessel disease is a consequence of systemic inflammation ( $\mathrm{Gu}$ et al., 2018a). Small vessel disease (SVD) refers to any pathologic process that damages small end arteries, arterioles, venules, and capillaries (Poggesi et al., 2012). Rouhl et al. (2012) confirmed the activation of an inflammatory process in 163 patients suffering their firstever lacunar stroke, and this was followed a few short years later by a study in patients who suffered traumatic brain injury which confirmed that acute cerebral blood flow was a biomarker of underlying neuroinflammatory pathology (Sankar et al., 2019). Thus, inflammatory mediators are mechanistically associated with both SVD and reduced CBF.

Vermeer et al. (2007) included obesity in the list of lifestyles that are associated with SVD in the brain, evidenced on MRI by white matter hyperintensities. This association has been confirmed by other investigators (Yamashiro et al., 2014). Elevated BMI is also known to be associated with decreased 


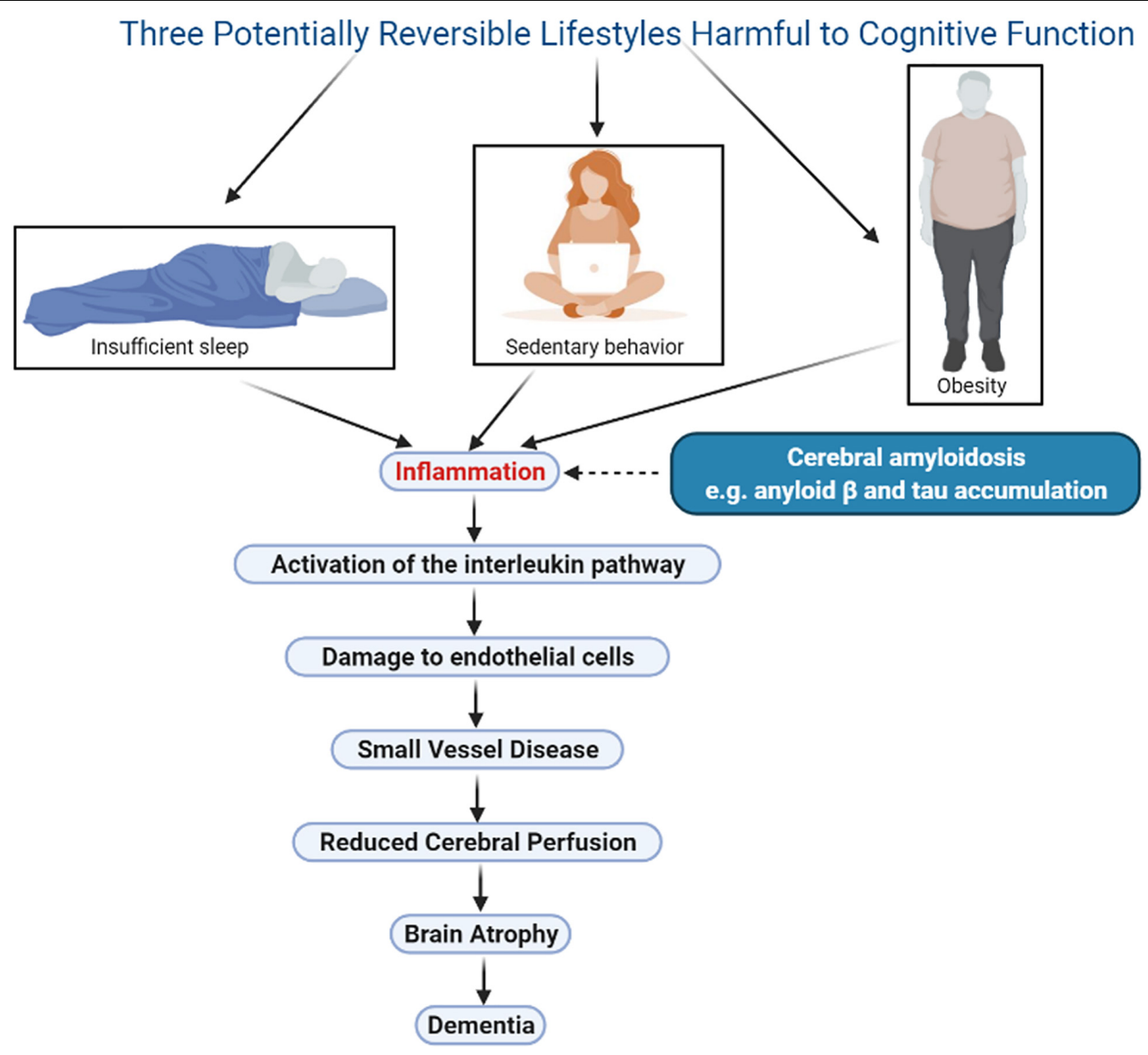

FIGURE 1 | Illustration of a unifying hypothesis that proposes lifestyles that negatively impact cognition do so through the same sequence of events: inflammation, activation of the interleukin pathway, small vessel disease, damage to endothelial cells, decline in cerebral perfusion, and brain atrophy. Three harmful lifestyles are shown, obesity, sedentary behavior and insufficient sleep, all of which are potentially reversible therefore offering an opportunity for meaningful therapeutic interventions. Cerebral amyloidosis may play a synergistic role in this sequence however this phenomenon is currently not reversible. Figure was made in BioRender.com.

blood flow in the prefrontal cortex of otherwise healthy adults (Willeumier et al., 2011), and a more recent evaluation using single photon emission computed tomography (SPECT) showed that higher BMI correlated with decreased perfusion in virtually all brain regions (Amen et al., 2020). Perhaps not surprisingly, given these facts, obese individuals had lower total cerebral brain volume compared to metabolically healthy non-obese individuals (Angoff et al., 2019), and Hamer and Batty (2019) reported that higher levels of all obesity measures were related to lower gray matter volume in many brain regions, an observation confirmed by Dekkers et al. (2019). Stanek et al. (2011) reported that a "(BMI $\mathrm{X}$ age") interaction was noted in the corpus callosum, implying that damage to certain brain structures increases with both the degree of obesity and its duration.

In conclusion, obesity as a risk factor for cognitive decline would seem to fit well into the working hypothesis proposed here, namely that a specific physiological sequence, triggered by inflammation and resulting ultimately in brain atrophy, underlies the progression from the risk factor to the eventual cognitive decline.

\section{SEDENTARY LIFESTYLE}

Sedentary behavior refers to activities that do not substantially increase energy expenditure above the resting level (Pate et al., 2008). In 2011, Barnes and Yaffe (2011) projected the impact of risk factor reduction on the prevalence of dementia and proposed that in North America, 21\% of dementias could be attributed to physical inactivity. More recently, a study in Swedish women showed that $32 \%$ of those with low peak fitness, $25 \%$ with medium and 5\% with high fitness subsequently developed dementia (Horder et al., 2018). In a systematic review and meta-analysis, Sanders et al. (2019) suggested a doseresponse relationship between exercise and cognitive function, confirming the conclusion from a 10 -year follow-up study by 
Hamer et al. (2018) which showed that memory and executive function were preserved by physical activity.

There is abundant literature confirming that sedentary behavior is associated with an inflammatory environment, and that exercise has anti-inflammatory benefits. After adjusting for all potential extraneous factors, sedentary behavior was associated with higher levels of TNF $\alpha$ and leptin (Allison et al., 2012). Henson et al. (2013) showed that sedentary time was detrimentally associated with levels of CRP, IL-6, and leptin. This was followed by a study in which reallocating 30 min of sedentary time to moderate and vigorous physical activity was associated with a more favorable inflammatory profile, characterized by higher adiponectin and lower complement component $\mathrm{C} 3$, leptin, and IL-6 (Phillips et al., 2017). Parsons et al. (2017) confirmed in the same year that sedentary activity was associated with higher levels of IL-6, CRP, and tissue plasminogen activator (tPA), while higher total physical activity levels were associated with lower levels of these inflammatory mediators. In 2018, Tsai et al. (2017) reported that an acute bout of aerobic exercise significantly increased serum levels of brain derived neurotrophic factor (BDNF) and insulin-like growth factor 1 (IGF-1), which may counter the inflammatory environment.

The literature linking prolonged sitting with a decline in cerebral perfusion is extensive. Carter et al. (2018) reported a significant decline in blood flow velocity in the middle cerebral artery when sitting is uninterrupted, compared with 2 -min light intensity walking breaks every $30 \mathrm{~min}$. The benefit disappeared if the sitting period was prolonged. Zlatar et al. (2019) confirmed that longer sedentary time was significantly associated with lower $\mathrm{CBF}$ in lateral and medial frontal regions, which confirmed the earlier work by Bailey et al. (2013) showing that sedentary aging is associated with a longitudinal decline in CBF. Finally, the literature has persistently confirmed the positive impact of regular physical activity on CBF (Kleinloog et al., 2019; Hartman et al., 2020; Maasakkers et al., 2020; Steventon et al., 2020).

White matter hyperintensity volume in the brain is higher with longer sedentary time, and the relationship appears to be modulated by kidney function (Bronas et al., 2019). Rhyu et al. (2010) showed in monkeys that exercise increased the vascular volume fraction in the motor cortex, a response that disappeared after a 3-month sedentary period. Burzynska et al. (2016) reported that moderate to vigorous physical activity was associated with lower white matter hyperintensity volume in healthy older adults, and a randomized controlled trial in older women showed that resistance training reduced the progression in white matter hyperintensity (Bolandzadeh et al., 2015).

The association of sedentary behavior with brain atrophy is also amply evidenced in the literature. Siddarth et al. (2018) reported that the thickness of the medial temporal lobe, the parahippocampal and entorhinal regions and subiculum correlated inversely with hours of sitting per day. Hashimoto et al. (2016) investigated the relationship between physical activity and hippocampal volume and concluded that hippocampal atrophy was associated with physical inactivity. A review by Cooper et al. (2018) confirmed that running improves hippocampal neurogenesis, neural circuitry, and synaptic plasticity. In a sub-sample from the Reykjavik Study cohort, Arnardottir et al. (2016) showed that more gray and white matter volumes at baseline were associated with more total physical activity, and the 5-year change in MRI-derived white matter volume was associated with total physical activity. The authors summarize their findings by confirming that a robust association exists between brain atrophy and lack of physical activity.

\section{SLEEP INSUFFICIENCY}

Sleep is essential, and enough of it is crucial for cognitive function. The brain uses sleep as an opportunity to rid itself of metabolites that accumulated during wakefulness (Xie et al., 2013), repair connections that may have been damaged (Bellesi et al., 2013), and consolidate learning acquired during the day (Yang et al., 2014).

Many environmental factors can interfere with the adequacy of sleep, including the use of technology (Gamble et al., 2014), the consumption of stimulants (Ogeil and Phillips, 2015), and environmental noise (Hume et al., 2012). Short sleep duration and disturbed sleep, when chronic, are related to several cardiovascular (Huang L.-K. et al., 2020; Huang T. et al., 2020), metabolic and neuropsychiatric diseases (Irwin, 2015). As well, a number of medical conditions can render sleep inadequate, most prominently sleep apnea (Dempsey et al., 2010), and restless leg syndrome (Guo et al., 2017). In studying the impact of sleep disturbances on inflammation or small vessel disease, it is important but often difficult to separate the contribution made by any underlying medical condition from that made by the associated sleep derangement. For example, obstructive sleep apnea does indeed impact sleep adequacy, but it also triggers hypoxia, and it becomes difficult but important to take that into account when studying the impact of sleep on cognition.

With these limitations in mind, there is ample evidence that poor sleep quality is associated with a reduction in cognitive functions (Lim et al., 2013; Osorio et al., 2015). Sleep promotes the formation of dendritic spines to consolidate learning into long-term memory (Yang et al., 2014), and poor sleep quality and duration interrupt this process. Adequate sleep also promotes inflammatory homeostasis and by contrast, insufficient sleep activates several inflammatory mediators and can lead to systemic and chronic low-grade inflammation (Besedovsky et al., 2019). Sleep duration of less than $5 \mathrm{~h}$ elevates the body's inflammatory burden including elevations in CRP, IL-6 and TNF $\alpha$ (Smagula et al., 2016). Disturbed sleep and insomnia are also strongly associated with upregulation of CRP and IL-6 (Irwin et al., 2016). Adolescents are not spared: compared to controls, those suffering from insomnia and short sleep duration exhibit systemic inflammation and significantly higher CRP level (FernandezMendoza et al., 2017; Slavish et al., 2018). In a systematic review of the topic, extreme long sleep duration as well as sleep disturbance and shorter sleep duration were associated with higher levels of CRP and IL-6 (Irwin et al., 2016). Telomere length is an index of cellular aging, and since ongoing inflammation is known to contribute to telomere dysfunction (Jose et al., 2017), it is not surprising that shortened sleep has been shown to 
contribute to shortening of the telomere (Kaszubowska, 2008; Childs et al., 2015).

Brain atrophy is evident in those who are perennially sleep deprived (Sexton et al., 2014; Gelber et al., 2015). This is presumed to be a consequence of small vessel disease, and several studies have shown an association between poor sleep quality and the severity of white matter hyperintensities noted on MRI (Ramos et al., 2014; Del Brutto et al., 2015). Each hour of reduced sleep duration significantly augmented the expansion rate of the ventricles (Lo et al., 2014). Fjell et al. (2020) correlated self-reported sleep measures with MRI-derived hippocampal volumes in more than 3000 cognitively normal participants and reported that worse sleep characteristics correlated with greater hippocampal volume loss. In restless leg syndrome, a major cause of sleep disturbance, the longer the duration of the condition the more likely it was to be accompanied by brain small vessel disease (Ferri et al., 2016), and obstructive sleep apnea, when moderate to severe, has also been reported as an independent risk factor for the development of cerebral white matter changes (Kim et al., 2013).

The impact of sleep disturbances on CBF has been studied in acute sleep deprivation and in the setting of sleep apnea. Regional cerebral perfusion was reduced after acute sleep deprivation (Zhou et al., 2019), and patients with insomnia showed a consistent pattern of hypoperfusion across 8 pre-selected cerebral regions, with deactivation in the basal ganglia being particularly pronounced (Smith et al., 2002). In untreated obstructive sleep apnea, $\mathrm{CBF}$ was significantly lower in multiple brain regions, and the hypoperfusion partially reversed when treatment of the apnea was instituted (Kim et al., 2017). Using SPECT, Joo et al. (2007) showed that in patients suffering with obstructive sleep apnea regional $\mathrm{CBF}$ was reduced in bilateral hippocampal gyri and other regions involved in memory function, spatial learning, executive function, and attention. This association of sleep disturbance with reduced $\mathrm{CBF}$ in brain regions essential for cognitive functions has subsequently been confirmed (Baril et al., 2015).

\section{DISCUSSION}

The 2020 Lancet Commission Report lists 12 modifiable risk factors that are associated with cognitive decline. The Report states that together these account for $40 \%$ of worldwide dementias (Livingston et al., 2020). The report also emphasizes that the number of people with dementia is rising and stresses the importance of considering a life course model for these modifiable risk factors. The report gives the example of weight and blood pressure, which usually fall in later life, and warns that lower weight in later life might signify illness, not an absence of risk.

The three lifestyles chosen for discussion here were included in the Lancet Commission report as contributing to dementia. Their prevalence in society is increasing. Particularly in western society the populations are becoming more obese (Mitchell et al., 2011), more sedentary (Owen et al., 2010), and the demands of work and other responsibilities frequently interfere with the adequacy of sleep (Bixler, 2009). Taking these 3 lifestyles as prototypes for those that contribute to a decline in cognition, a sequence of events is proposed to explain how a harmful lifestyle leads to this unfortunate outcome.

The hypothesis offered suggests that the offending lifestyle leads to the persistent generation of inflammatory mediators that are harmful to the viability of small vessels. Several articles have confirmed the link between small vessel disease and the white matter hyperintensities seen on MRI (Verhaaren et al., 2015; Wardlaw et al., 2015; Rosenberg et al., 2016; ter Telgte et al., 2018). It is proposed that small vessel disease eventually leads to a decline in CBF, followed by atrophy of cerebral structures essential for cognitive function. Our hypothesis does not necessarily imply the activation of neuroinflammation but may lead to it subsequently (Low et al., 2021).

The pathophysiologic sequence of events proposed here is evident with each of the harmful lifestyles selected, but the hypothesis that this sequence of events occurs with every harmful lifestyle will only be confirmed when longitudinal studies make the relevant measurements, in the same population, starting with cognitively normal individuals and following them with simultaneous periodic measurements of cognitive function, inflammation profile, the viability of cerebral small vessels, determination of regional $\mathrm{CBF}$, and measurement of the volume of brain structures relevant to cognitive function. The success of any interventional studies to modify the course of dementia can only be judged against this information.

Ever since the description of the extracellular amyloid plaques and the intracellular tau neurofibrillary tangles accumulating in the brains of Alzheimer patients, the therapeutic emphasis has been on eliminating them from the brain. Several drug trials have now been completed which targeted these proteins without significant favorable impact on the cognitive deficits of the patients (Yiannopoulou et al., 2019). Nonetheless, cerebral amyloidosis and other causes of inflammation can have synergistic deleterious effects on cognitive function. To this date however, the only effective approach to avoiding or delaying dementia may be the correction of lifestyles known to contribute to cognitive decline.

In a major study made possible by the Alzheimer Disease Neuroimaging Initiative (ADNI), which collected information on several brain parameters starting with cognitively normal patients and following them as their cognitive functions declined, it was shown that these proteins accumulated late, following several physiological events in the brain, with the initial trigger to the entire cascade being a decline in $\mathrm{CBF}$, an event that preceded the appearance of the clinical syndrome by years (Iturria-Medina et al., 2016). The hypothesis presented here is further upstream from the decline in CBF and seeks to link lifestyles known to be harmful to cognition into current accepted knowledge.

To explain the link between dementia risk factors and the reduction in CBF, investigators have explored the longitudinal relationship between the presence of cerebral small vessel disease and the decline in CBF, particularly with a view to determining which is cause and which is effect. Shi et al. (2016) performed a systematic review of studies that assessed CBF in small vessel disease, and their results suggest that hypoperfusion in the whole brain and low cortical blood flow is likely a consequence of 
white matter hyperintensities. Van der Veen et al. (2015) had a year earlier shown that larger periventricular and deep white matter hyperintensities were associated with a decline in $\mathrm{CBF}$ 4 years later, but reduced baseline CBF was not associated with progression of lacunes and white matter hyperintensities. The consensus then appears to be that small vessel disease (SVD) precedes the decline in CBF, lending support to the hypothesis proposed here.

Endothelial cells form the main barrier between the circulating blood and the vessel wall, and they are the primary target of inflammation (Steyers and Miller, 2014). Inflammatory markers have been associated with periventricular white matter hyperintensities, the main MRI signature of SVD in the brain (Rosano et al., 2012). Several abnormalities in the interleukin pathways have been linked to SVD including TNF $\alpha$, IL-10, and IL-21 (Swardfager et al., 2017). Flex et al. (2014) had linked proinflammatory gene polymorphisms to dementia, and C-reactive protein is considered an endothelial toxin that is predictive of SVD (Mitaki et al., 2016). It is therefore no surprise that midlife systemic inflammation has been associated with the appearance of SVD in later life (Walker et al., 2017).

The idea that harmful lifestyles lead to inflammation was emphasized by a number of investigators, most prominently by Littman a number of years ago (Littman et al., 2007), and more recently by Newcombe et al. (2018), and then Nivukoski et al. (2019).

The link between inflammation and subsequent dementia extends to systemic conditions associated with inflammation, including inflammatory bowel disease (Zhang et al., 2020), and rheumatoid arthritis (Sangha et al., 2020), both of which have been associated with a higher dementia risk. This led to several attempts to treat Alzheimer's disease by targeting neuroinflammation (Ardura-Fabregat et al., 2017), but none were sufficiently promising to merit further assessment.

If the hypothesis offered here is to be truly universal, each of the 12 harmful lifestyles identified in the Lancet Report should be shown to be associated with inflammation and its consequences as proposed here. That is beyond the scope of this review, but inflammation is known to occur in most dementia risk factors identified by the Lancet Report, including excessive alcohol intake, smoking, depression, diabetes, and air pollution. Less education, identified as a risk factor for dementia, may lead to poverty which may lead to SVD through the more affordable pro-inflammatory fat-laden and heavily processed diet.

One cognitive risk factor identified in the Lancet Report that may not at first glance fit into the proposed hypothesis is hearing deficit. Having said that, midlife hearing impairment is associated with temporal lobe volume loss including in the hippocampus (Armstrong et al., 2019). Eckert et al. (2013) have used an estimate of SVD to show that agerelated changes in low frequency hearing was related to a global decline in vascular health, and Tabuchi (2014) has described the occurrence of auditory dysfunction in patients with cerebrovascular disease. Hearing deficit may also accompany dementia risk factors such as obesity ( $\mathrm{Hu}$ et al., 2020), sedentary behavior (Loprinzi, 2013), and sleep apnea (Chopra et al., 2016). Hearing loss therefore may be a consequence of inflammation, and evidence that inflammation-induced vasospasm is involved in the pathogenesis of acquired sensorineural hearing loss was eloquently presented recently by Eisenhut (2019). Windsor and Ruckenstein (2018), in fact suggested that anti-inflammatory therapies may reduce sensorineural hearing loss. In addition, a study by Ponticorvo et al. (2019) showed that there was reduced cerebral perfusion in patients with hearing loss detectable prior to brain structural damage. This is not to deny that the disconnection from the auditory environment that hearing deficit implies may also play a role in inducing cognitive deficit, since those using hearing aids reduce their risk for cognitive decline (Amieva et al., 2018; Maharani et al., 2018), but hearing deficit may fit into the hypothesis presented here better than suspected.

The hypothesis presented raises the need for individual, societal and pharmacological responses that could reduce the risk of dementia. Disseminating the message that dementia is not inevitable as we get older, and that individuals have significant control over their dementia risk, can be very empowering and may lead to individuals incorporating lifestyles and habits that reduce the risk. A concerted effort to spread this message by the organizations implicated in dementia prevention and care can be effective.

It is important in this regard to be reminded that the sequence of pathophysiologic steps identified here are at play for years prior to the appearance of cognitive deficits (Low et al., 2019). Consequently, it is unlikely that dementia would be alleviated by incorporating healthy lifestyles after the appearance of cognitive deficits. For this reason, younger populations should be targeted by the message that we individually have control over our risk for dementia as we age.

It is estimated that the cost of dementia care will overwhelm many healthcare budgets (World Health Organization, 2017). The determination of governments to reduce the prevalence of dementia should lead them to increase public education on the topic, impose taxation on unhealthy dietary drinks and foods, and stand the ground when industry responds. When we became convinced that smoking was the cause of most lung cancers, the healthcare system developed a discourse with the public and with governments which eventually led successfully to limitations on consumption. We need to regenerate that energy to reduce the risk of dementia in society.

Could an anti-inflammatory approach be useful in treating or delaying dementia? This review would suggest that the answer is positive, but clinical trials with anti-inflammatory drugs have found no evidence for efficacy in treating dementia (Rivers-Auty et al., 2020). There are multiple probable reasons for this, in addition to the time displacement between inflammation and its cognitive consequence. Inflammation is complex, and each lifestyle contributing to increased dementia risk may activate one or more specific inflammatory mediators requiring specific inhibitory intervention. Further research into the inflammatory profile associated with each dementia risk factor is needed, followed by the development and testing of an inhibitor. In the meantime, acquisition of healthy lifestyles may be the only path 
to reducing dementia risk for the individual and prevalence of the condition in society.

\section{CONCULSION}

This review proposes a unifying hypothesis that all lifestyles that contribute to cognitive impairment do so in the same stepwise fashion. The harmful lifestyle activates inflammatory mediators which cause cerebral small vessel disease. This then negatively impacts blood flow to the affected region causing it to lose volume and reducing its contribution to cognitive function. The review confirms this sequence in 3 lifestyles: obesity, sedentary existence and sleep insufficiency. It then shows that the same process likely occurs in hearing deficit. If confirmed, this

\section{REFERENCES}

Allison, M. A., Jensky, N. E., Marshall, S. J., Bertoni, A. G., and Cushman, M. (2012). Sedentary behavior and adiposity-associated inflammation: the MultiEthnic Study of Atherosclerosis. Am J Prev Med. 42, 8-13. doi: 10.1016/j. amepre.2011.09.023

Amen, D. G., Wu, J., George, N., and Newberg, A. (2020). Patterns of Regional Cerebral Blood Flow as a Function of Obesity in Adults. Journal of Alzheimer's Disease. 77, 1331-1337. doi: 10.3233/JAD-200655

Amieva, H., Ouvrard, C., Meillon, C., Rullier, L., and Dartigues, J. F. (2018). Death, Depression, Disability, and Dementia Associated With Self-reported Hearing Problems: A 25-Year Study. J Gerontol A Biol Sci Med Sci. 73, 1383-1389. doi: $10.1093 /$ gerona/glx250

Angoff, R., Himali, J. J., Ramachandran, V. S., Seshadri, S., Beiser, A., and Tsao, C. (2019). Relations of Metabolic Health and Obesity to Brain Aging in Young to Middle-Aged Adults. Circulation 140, A14734.

Ardura-Fabregat, A., Boddeke, E. W. G. M., Boza-Serrano, A., Brioschi, S., CastroGomez, S., Ceyzériat, K., et al. (2017). Targeting Neuroinflammation to Treat Alzheimer's Disease. CNS Drugs. 31, 1057-1082. doi: 10.1007/s40263-0170483-3

Armstrong, N. M., An, Y., Doshi, J., Erus, G., Ferrucci, L., Davatzikos, C., et al. (2019). Association of Midlife Hearing Impairment With Late-Life Temporal Lobe Volume Loss. JAMA Otolaryngol Head Neck Surg. 3, e191610. doi: 10. 1001/jamaoto.2019.1610

Arnardottir, N. Y., Koster, A., Domelen, D. R. V., Brychta, R. J., Caserotti, P., Eiriksdottir, G., et al. (2016). Association of change in brain structure to objectively measured physical activity and sedentary behavior in older adults: Age, Gene/Environment Susceptibility-Reykjavik Study. Behavioural Brain Research. 296, 118-124. doi: 10.1016/j.bbr.2015.09.005

Bailey, D. M., Marley, C. J., Brugniaux, J. V., Hodson, D., New, K. J., Ogoh, S., et al. (2013). Elevated aerobic fitness sustained throughout the adult lifespan is associated with improved cerebral hemodynamics. Stroke. 44, 3235-3238. doi: 10.1161/STROKEAHA.113.002589

Baril, A. A., Gagnon, K., Arbour, C., Soucy, J. P., Montplaisir, J., Gagnon, J. F., et al. (2015). Regional Cerebral Blood Flow during Wakeful Rest in Older Subjects with Mild to Severe Obstructive Sleep Apnea. Sleep. 38, 1439-1449. doi: $10.5665 /$ sleep.4986

Barnes, D. E., and Yaffe, K. (2011). The projected effect of risk factor reduction on Alzheimer's disease prevalence. Lancet Neurol. 10, 819-828. doi: 10.1016/ S1474-4422(11)70072-2

Bellesi, M., Pfister-Genskow, M., Maret, S., Keles, S., Tononi, G., and Cirelli, C. (2013). Effects of sleep and wake on oligodendrocytes and their precursors. $J$ Neurosci. 33, 14288-14300. doi: 10.1523/JNEUROSCI.5102-12.2013

Beltrán-Sánchez, H., Soneji, S., and Crimmins, E. M. (2015). Past, Present, and Future of Healthy Life Expectancy. Cold Spring Harb Perspect Med. 5, a025957. doi: 10.1101/cshperspect.a025957 hypothesis suggests individual, societal and therapeutic approaches that may counteract the growing burden of cognitive decline.

\section{AUTHOR CONTRIBUTIONS}

$\mathrm{AH}$ reviewed the literature and wrote the article.

\section{ACKNOWLEDGMENTS}

The author would like to acknowledge his gratitude to the University of Ottawa Brain and Mind Research Institute, and to Sarah Schock, for their support and contributions in the production of this manuscript.

Besedovsky, L., Lange, T., and Haack, M. (2019). The Sleep-Immune Crosstalk in Health and Disease. Physiol Rev. 99, 1325-1380. doi: 10.1152/physrev.00010. 2018

Bixler, E. (2009). Sleep and society: an epidemiological perspective. Sleep Med. 10 (Suppl. 1), S3-S6. doi: 10.1016/j.sleep.2009.07.005

Bolandzadeh, N., Tam, R., Handy, T. C., Nagamatsu, L. S., Hsu, C. L., Davis, J. C., et al. (2015). T. Resistance Training and White Matter Lesion Progression in Older Women: Exploratory Analysis of a 12-Month Randomized Controlled Trial. J Am Geriatr Soc. 63, 2052-2060. doi: 10.1111/jgs.13644

Bronas, U. G., Steffen, A., Dion, C., Boots, E. A., Arfanakis, K., Marquez, D. X., et al. (2019). Sedentary Time and White Matter Hyperintensity Volume in Older Adults. Med Sci Sports Exerc. 51, 1613-1618. doi: 10.1249/MSS. 0000000000001957

Burzynska, A. Z., Wong, C. N., Chaddock-Heyman, L., Olson, E. A., Gothe, N. P., Knecht, A., et al. (2016). White matter integrity, hippocampal volume, and cognitive performance of a world-famous nonagenarian track-and-field athlete. Neurocase. 22, 135-144. doi: 10.1080/13554794.2015.1074709

Carter, S. E., Draijer, R., Holder, S. M., Brown, L., Thijssen, D., and Hopkins, N. D. (2018). Regular walking breaks prevent the decline in cerebral blood flow associated with prolonged sitting. Journal of applied physiology 125, 790-798. doi: 10.1152/japplphysiol.00310.2018

Chait, A., and Hartigh, L. J. D. (2020). Adipose Tissue Distribution, Inflammation and Its Metabolic Consequences, Including Diabetes and Cardiovascular Disease. Front Cardiovasc Med. 7:22. doi: 10.3389/fcvm.2020.00022

Childs, B. G., Durik, M., Baker, D. J., and van Deursen, J. M. (2015). Cellular senescence in aging and age-related disease: from mechanisms to therapy. Nat Med. 21, 1424-1435. doi: 10.1038/nm.4000

Chopra, A., Jung, M., Kaplan, R. C., Appel, D. W., Dinces, E. A., Dhar, S., et al. (2016). Sleep Apnea Is Associated with Hearing Impairment: The Hispanic Community Health Study/Study of Latinos. J Clin Sleep Med. 12, 719-726. doi: $10.5664 /$ jcsm. 5804

Cooper, C., Moon, H. Y., and van Praag, H. (2018). On the Run for Hippocampal Plasticity. Cold Spring Harb Perspect Med. 8, a029736. doi: 10.1101/cshperspect. a029736

Dekkers, I. A., Jansen, P. R., and Lamb, H. J. (2019). Obesity, Brain Volume, and White Matter Microstructure at MRI: A Cross-sectional UK Biobank Study. Radiology. 292, 270. doi: 10.1148/radiol.2019194010

Del Brutto, O. H., Mera, R. M., Zambrano, M., Lama, J., Del Brutto, V. J., and Castillo, P. R. (2015). Poor sleep quality and silent markers of cerebral small vessel disease: a population-based study in community-dwelling older adults (The Atahualpa Project). Sleep Med. 16, 428-431. doi: 10.1016/j.sleep.2014. 10.023

Dempsey, J. A., Veasey, S. C., Morgan, B. J., and O'Donnell, C. P. (2010). Pathophysiology of sleep apnea [published correction appears in Physiol Rev.2010;90(2):797-8]. Physiol Rev. 90, 47-112. doi: 10.1152/physrev.00043. 2008 
Eckert, M. A., Kuchinsky, S. E., Vaden, K. I., Cute, S. L., Spampinato, M. V., and Dubno, J. R. (2013). White Matter Hyperintensities Predict Low Frequency Hearing in Older Adults. J Assoc Res Otolaryngol. 14, 425-433. doi: 10.1007/ s10162-013-0381-4

Eisenhut, M. (2019). Evidence Supporting the Hypothesis That InflammationInduced Vasospasm Is Involved in the Pathogenesis of Acquired Sensorineural Hearing Loss. Int J Otolaryngol. 2019, 4367240. doi: 10.1155/2019/4367240

Fernandez-Mendoza, J., Baker, J. H., Vgontzas, A. N., Gaines, J., Liao, D., and Bixler, E. O. (2017). Insomnia symptoms with objective short sleep duration are associated with systemic inflammation in adolescents. Brain Behav Immun. 61, 110-116. doi: 10.1016/j.bbi.2016.12.026

Ferri, R., Cosentino, F. I., Moussouttas, M., Lanuzza, B., Aricò, D., Bagai, K., et al. (2016). Silent Cerebral Small Vessel Disease in Restless Legs Syndrome. Sleep. 39, 1371-1377. doi: 10.5665/sleep.5966

Fjell, A. M., Sørensen, Ø, Amlien, I. K., Bartrés-Faz, D., Bros, D. M., Buchmann, N., et al. (2020). Self-reported sleep relates to hippocampal atrophy across the adult lifespan: results from the Lifebrain consortium. Sleep 43, zsz280. doi: 10.1093/sleep/zsz280

Flegal, K. M., Carroll, M. D., Kit, B. K., and Ogden, C. L. (2012). Prevalence of obesity and trends in the distribution of body mass index among US adults, 1999-2010. JAMA 307, 491-497. doi: 10.1001/jama.2012.39

Flex, A., Giovannini, S., Biscetti, F., Liperoti, R., Spalletta, G., Straface, G., et al. (2014). Effect of proinflammatory gene polymorphisms on the risk of Alzheimer's disease. Neurodegener Dis. 13, 230-236. doi: 10.1159/0003 53395

Ford, E. S., Maynard, L. M., and Li, C. (2014). Trends in mean waist circumference and abdominal obesity among US adults, 1999-2012. JAMA. 312, 1073-1075. doi: 10.1001/jama.2014.8362

Gamble, A. L., D’Rozario, A. L., Bartlett, D. J., Williams, S., Bin, Y. S., Grunstein, R. R., et al. (2014). Adolescent sleep patterns and night-time technology use: results of the Australian Broadcasting Corporation's Big Sleep Survey. PLoS One 9:e111700. doi: 10.1371/journal.pone.0111700

GBD 2016 Dementia Collaborators. (2019). Global, regional, and national burden of Alzheimer's disease and other dementias, 1990-2016: a systematic analysis for the Global Burden of Disease Study 2016. Lancet Neurol. 18, 88-106. doi: 10.1016/S1474-4422(18)30403-4

Gelber, R. P., Redline, S., Ross, G. W., Petrovitch, H., Sonnen, J. A., Zarow, C., et al. (2015). Associations of brain lesions at autopsy with polysomnography features before death. Neurology. 84, 296-303. doi: 10.1212/WNL.0000000000001163

Gu, Y., Gutierrez, J., Meier, I. B., Guzman, V. A., Manly, J. J., Schupf, N., et al. (2018a). Circulating inflammatory biomarkers are related to cerebrovascular disease in older adults. Neurol Neuroimmunol Neuroinflamm. 6, e521. doi: 10.1212/NXI.0000000000000521

Gu, Y., Manly, J. J., Mayeux, R. P., and Brickman, A. M. (2018b). An Inflammationrelated Nutrient Pattern is Associated with Both Brain and Cognitive Measures in a Multiethnic Elderly Population. Current Alzheimer Research 15, 493-501. doi: $10.2174 / 1567205015666180101145619$

Guo, S., Huang, J., Jiang, H., Han, C., Li, J., Xu, X., et al. (2017). Restless Legs Syndrome: From Pathophysiology to Clinical Diagnosis and Management. Front Aging Neurosci. 9:171. doi: 10.3389/fnagi.2017.00171

Hamer, M., and Batty, G. D. (2019). Association of body mass index and waistto-hip ratio with brain structure: UK Biobank study. Neurology. 92, e591-e600. doi: 10.1212/WNL.0000000000006879

Hamer, M., Terrera, G. M., and Demakakos, P. (2018). Physical activity and trajectories in cognitive function: English Longitudinal Study of Ageing. $J$ Epidemiol Community Health. 72, 477-483. doi: 10.1136/jech-2017-210228

Hartman, Y. A. W., Tillmans, L. C. M., Benschop, D. L., Hermans, A. N. L., Nijssen, K. M. R., Eijsvogels, T. M. H., et al. (2020). Long-Term and Acute Benefits of Reduced Sitting on Vascular Flow and Function. Med Sci Sports Exerc. 53, 341-350. doi: 10.1249/MSS.0000000000002462

Hashimoto, M., Araki, Y., Takashima, Y., Nogami, K., Uchino, A., Yuzuriha, T., et al. (2016). Hippocampal atrophy and memory dysfunction associated with physical inactivity in community-dwelling elderly subjects: The Sefuri study. Brain Behav. 7, e00620. doi: 10.1002/brb3.620

Henson, J., Yates, T., Edwardson, C. L., Khunti, K., Talbot, D., Gray, L. J., et al. (2013). Sedentary Time and Markers of Chronic Low-Grade Inflammation in a High Risk Population. PLoS One. 8:e78350. doi: 10.1371/journal.pone.00 78350
Horder, H., Johansson, L., Guo, X., Grimby, G., Kern, S., Östling, S., et al. (2018). Midlife cardiovascular fitness and dementia: a 44-year longitudinal population study in women. Neurology. 90, e1298-e1305. doi: 10.1212/WNL. 0000000000005290

Hu, H., Tomita, K., Kuwahara, K., Yamamoto, M., Uehara, A., Kochi, T., et al. (2020). Obesity and risk of hearing loss: A prospective cohort study. Clin Nutr. 39, 870-875. doi: 10.1016/j.clnu.2019.03.020

Huang, L.-K., Chao, S.-P., and Hu, C.-J. (2020). Clinical trial of new drugs for Alzheimer disease. J Biomed Sci 27, 18. doi: 10.1186/s12929-019-0609-7

Huang, T., Mariani, S., and Redline, S. (2020). Sleep Irregularity and Risk of Cardiovascular Events: The Multi-Ethnic Study of Atherosclerosis. J Am Coll Cardiol. 75, 991-999. doi: 10.1016/j.jacc.2019.12.054

Hume, K. I., Brink, M., and Basner, M. (2012). Effects of environmental noise on sleep. Noise \& Health. 14, 297-302. doi: 10.4103/1463-1741.104897

Irwin, M. R. (2015). Why sleep is important for health: a psychoneuroimmunology perspective. Annu Rev Psychol. 66, 143-172. doi: 10.1146/annurev-psych010213-115205

Irwin, M. R., Olmstead, R., and Carroll, J. E. (2016). Sleep Disturbance, Sleep Duration, and Inflammation: A Systematic Review and Meta-Analysis of Cohort Studies and Experimental Sleep Deprivation. Biol Psychiatry. 80, 40-52. doi: 10.1016/j.biopsych.2015.05.014

Iturria-Medina, Y., Sotero, R., Toussaint, P., Mateos-Pérez, J. M., and Evans, A. C. (2016). Early role of vascular dysregulation on late-onset Alzheimer's disease based on multifactorial data-driven analysis. Nat Commun. 11934, 1-14. doi: 10.1038/ncomms 11934

Joo, E. Y., Tae, W. S., Han, S. J., Cho, J. W., and Hong, S. B. (2007). Reduced cerebral blood flow during wakefulness in obstructive sleep apnea-hypopnea syndrome. Sleep. 30, 1515-1520. doi: 10.1093/sleep/30.11.1515

Jose, S. S., Bendickova, K., Kepak, T., Krenova, Z., and Fric, J. (2017). Chronic Inflammation in Immune Aging: Role of Pattern Recognition Receptor Crosstalk with the Telomere Complex. Front Immunol. 8:1078. doi: 10.3389/ fimmu.2017.01078

Karunakaran, D., Turner, A. W., Duchez, A. C., Soubeyrand, S., Rasheed, A., Smyth, D., et al. (2020). RIPK1 gene variants associate with obesity in humans and can be therapeutically silenced to reduce obesity in mice. Nat Metab. 2, 1113-1125. doi: 10.1038/s42255-020-00279-2

Kaszubowska, L. (2008). Telomere shortening and ageing of the immune system. J Physiol Pharmacol. 59 (Suppl. 9), 169-186.

Kern, P. A., Ranganathan, S., Li, C., Wood, L., and Ranganathan, G. (2001). Adipose tissue tumor necrosis factor and interleukin-6 expression in human obesity and insulin resistance. Am J Physiol Endocrinol Metab. 280, E745-E751.

Kerti, L., Witte, A. V., Winkler, A., Grittner, U., Rujescu, D., and Flöel, A. (2013). Higher glucose levels associated with lower memory and reduced hippocampal microstructure. Neurology. 81, 1746-1752. doi: 10.1212/01.wnl.0000435561. 00234.ee

Kim, H., Yun, C. H., Thomas, R. J., Lee, S. H., Seo, H. S., Cho, E. R., et al. (2013). Obstructive sleep apnea as a risk factor for cerebral white matter change in a middle-aged and older general population. Sleep 36, 709B-715B. doi: 10.5665/ sleep. 2632

Kim, J. S., Seo, J. H., Kang, M. R., Seong, M. J., Lee, W. G., Joo, E. Y., et al. (2017). Effect of continuous positive airway pressure on regional cerebral blood flow in patients with severe obstructive sleep apnea syndrome. Sleep Med. 32, 122-128. doi: 10.1016/j.sleep.2016.03.010

Kleinloog, J. P. D., Mensink, R. P., Ivanov, D., Adam, J. J., Uludað, K., and Joris, P. J. (2019). Aerobic Exercise Training Improves Cerebral Blood Flow and Executive Function: A Randomized, Controlled Cross-Over Trial in Sedentary Older Men. Front Aging Neurosci. 11:333. doi: 10.3389/fnagi.2019. 00333

Laurin, D., Verreault, R., Lindsay, J., MacPherson, K., and Rockwood, K. (2001). Physical activity and risk of cognitive impairment and dementia in elderly persons. Arch Neurol. 58, 498-504. doi: 10.1001/archneur.58.3.498

Lim, A. S., Kowgier, M., Yu, L., Buchman, A. S., and Bennett, D. A. (2013). Sleep Fragmentation and the Risk of Incident Alzheimer's Disease and Cognitive Decline in Older Persons. Sleep. 36, 1027-1032. doi: 10.5665/sleep.2802

Littman, A. J., Vitiello, M. V., Foster-Schubert, K., Ulrich, C. M., Tworoger, S. S., Potter, J. D., et al. (2007). Sleep, ghrelin, leptin and changes in body weight during a 1-year moderate-intensity physical activity intervention. Int J Obes (Lond). 31, 466-475. doi: 10.1038/sj.ijo.0803438 
Livingston, G., Huntley, J., Sommerlad, A., Ames, D., Ballard, C., Banerjee, S., et al. (2020). Dementia prevention, intervention, and care: 2020 report of the Lancet Commission. Lancet. 396, 413-446. doi: 10.1016/S0140-6736(20)30367-6

Lo, J. C., Loh, K. K., Zheng, H., Sim, S. K., and Chee, M. W. (2014). Sleep duration and age-related changes in brain structure and cognitive performance. Sleep. 37, 1171-1178. doi: 10.5665/sleep. 3832

Loprinzi, P. D. (2013). Association between accelerometer-assessed sedentary behavior and objectively-measured hearing sensitivity in older US adults. Prev Med. 57, 143-145. doi: 10.1016/j.ypmed.2013.05.007

Low, A., Mak, E., Malpetti, M., Passamonti, L., Nicastro, N., Stefaniak, J. D., et al. (2021). In vivo neuroinflammation and cerebral small vessel disease in mild cognitive impairment and Alzheimer's disease. J Neurol Neurosurg \& Psych. 92, 45-52. doi: 10.1136/jnnp-2020-323894

Low, A., Mak, E., Rowe, J. B., Markus, H. S., and O’Brien, J. T. (2019). Inflammation and cerebral small vessel sidease: A systematic review. Ageing Res Rev. 53, 100916. doi: 10.1016/j.arr.2019.100916

Maasakkers, C. M., Melis, R. J. F., Kessels, R. P. C., Gardiner, P. A., Olde Rikkert, M. G. M., Thijssen, D. H. J., et al. (2020). The short-term effects of sedentary behaviour on cerebral hemodynamics and cognitive performance in older adults: a cross-over design on the potential impact of mental and/or physical activity. Alzheimers Res Ther. 12, 76. doi: 10.1186/s13195-02000644-z

Maharani, A., Dawes, P., Nazroo, J., Tampubolon, G., Pendleton, N., and SenseCog WP1 group. (2018). Longitudinal Relationship Between Hearing Aid Use and Cognitive Function in Older Americans. J Am Geriatr Soc. 66, 1130-1136. doi: $10.1111 /$ jgs. 15363

Makki, K., Froguel, P., and Wolowczuk, I. (2013). Adipose tissue in obesity-related inflammation and insulin resistance: cells, cytokines, and chemokines. ISRN Inflamm. 2013, 139239. doi: 10.1155/2013/139239

Martin, A. A., and Davidson, T. L. (2014). Human cognitive function and the obesogenic environment. Physiol Behav. 136, 185-193. doi: 10.1016/j.physbeh. 2014.02.062

Mitaki, S., Nagai, A., Oguro, H., and Yamaguchi, S. (2016). C-reactive protein levels are associated with cerebral small vessel-related lesions. Acta Neurol Scand 133, 68-74. doi: 10.1111/ane.12440

Mitchell, N. S., Catenacci, V. A., Wyatt, H. R., and Hill, J. O. (2011). Obesity: overview of an epidemic. Psychiatr Clin North Am. 34, 717-732. doi: 10.1016/j. psc.2011.08.005

Newcombe, E. A., Camats-Perna, J., Silva, M. L., Valmas, N., Huat, T. J., and Medeiros, R. (2018). Inflammation: the link between comorbidities, genetics, and Alzheimer's disease. J Neuroinflammation. 15, 276. doi: 10.1186/s12974018-1313-3

Nivukoski, U., Niemelä, M., Bloigu, A., Bloigu, R., Aalto, M., Laatikainen, T., et al. (2019). Impacts of unfavourable lifestyle factors on biomarkers of liver function, inflammation and lipid status. PLoS One. 14:e0218463. doi: 10.1371/journal. pone. 0218463

Ogeil, R. P., and Phillips, J. G. (2015). Commonly used stimulants: Sleep problems, dependence and psychological distress. Drug Alcohol Depend. 153, 145-151. doi: 10.1016/j.drugalcdep.2015.05.036

Osorio, R. S., Gumb, T., Pirraglia, E., Varga, A. W., Lu, S. E., Lim, J., et al. (2015). Sleep-disordered breathing advances cognitive decline in the elderly. Neurology. 84, 1964-1971. doi: 10.1212/WNL.0000000000001566

Owen, N., Sparling, P. B., Healy, G. N., Dunstan, D. W., and Matthews, C. E. (2010). Sedentary behavior: emerging evidence for a new health risk. Mayo Clin Proc. 85, 1138-1141. doi: $10.4065 / \mathrm{mcp} .2010 .0444$

Oxford, A. E., Stewart, E. S., and Rohn, T. T. (2020). Clinical Trials in Alzheimer's Disease: A Hurdle in the Path of Remedy. Int J Alzheimers Dis. 2020, 5380346. doi: $10.1155 / 2020 / 5380346$

Parsons, T. J., Sartini, C., Welsh, P., Sattar, N., Ash, S., Lennon, L. T., et al. (2017). Physical Activity, Sedentary Behavior, and Inflammatory and Hemostatic Markers in Men. Med Sci Sports Exerc. 49, 459-465. doi: 10.1249/MSS. 0000000000001113

Pate, R. R., O’Neill, J. R., and Lobelo, F. (2008). The Evolving Definition of "Sedentary". Exerc. Sport Sci. Rev. 36, 173-178. doi: 10.1097/JES. 0b013e3181877dla

Patterson, C. (2018). World Alzheimer report 2018. The state of the art of dementia research: New frontiers. London: Alzheimer's Disease International (ADI), London.
Phillips, C. M., Dillon, C. B., and Perry, I. J. (2017). Does replacing sedentary behaviour with light or moderate to vigorous physical activity modulate inflammatory status in adults? Int J Behav Nutr Phys Act. 14, 138. doi: 10.1186/ s12966-017-0594-8

Poggesi, A., Salvadori, E., Pantoni, L., Pracucci, G., Cesari, F., Chiti, A., et al. (2012). Risk and Determinants of Dementia in Patients with Mild Cognitive Impairment and Brain Subcortical Vascular Changes: A Study of Clinical Neuroimaging, and Biological Markers-The VMCI-Tuscany Study: Rationale, Design, and Methodology. Int J Alzheimers Dis. 2012, 608013.

Ponticorvo, S., Manara, R., Pfeuffer, J., Cappiello, A., Cuoco, S., Pellecchia, M. T., et al. (2019). Cortical pattern of reduced perfusion in hearing loss revealed by ASL-MRI. Hum Brain Mapp. 40, 2475-2487. doi: 10.1002/hbm. 24538

Qiu, C., and Fratiglioni, L. (2018). Aging without Dementia is Achievable: Current Evidence from Epidemiological Research. J Alzheimers Dis. 62, 933-942. doi: 10.3233/JAD- 171037

Qizilbash, N., Gregso, J., Johnson, M. E., Pearce, N., Douglas, I., Wing, K., et al. (2015). BMI and risk of dementia in two million people over two decades: a retrospective cohort study. Lancet Diabetes Endocrinol. 3, 431-436. doi: 10. 1016/S2213-8587(15)00033-9

Ramos, A. R., Dong, C., Rundek, T., Elkind, M. S., Boden-Albala, B., Sacco, R. L., et al. (2014). Sleep duration is associated with white matter hyperintensity volume in older adults: The Northern Manhattan Study. J Sleep Res. 23, 524-530. doi: 10.1111/jsr.12177

Rhyu, I. J., Bytheway, J. A., Kohler, S. J., Lange, H., Lee, K. J., Boklewski, J., et al. (2010). Effects of aerobic exercise training on cognitive function and cortical vascularity in monkeys. Neuroscience. 167, 1239-1248. doi: 10.1016/j. neuroscience.2010.03.003

Rivers-Auty, J., Mather, A. E., Peters, R., Lawrence, C. B., and Brough, D. (2020). Anti-inflammatories in Alzheimer's disease-potential therapy or spurious correlate? Brain Communications. 2, fcaa109. doi: 10.1093/braincomms/ fcaa 109

Rosano, C., Marsland, A. L., and Gianaros, P. J. (2012). Maintaining brain health by monitoring inflammatory processes: a mechanism to promote successful aging. Aging Dis. 3, 16-33.

Rosenberg, G. A., Wallin, A., Wardlaw, J. M., Markus, H. S., Montaner, J., Wolfson, L., et al. (2016). Consensus statement for diagnosis of subcortical small vessel disease. J Cereb Blood Flow Metab. 36, 6-25. doi: 10.1038/jcbfm. 2015.172

Rouhl, R. R., Damoiseaux, J. G. M. C., Lodder, J., Theunissen, R. O. M. F. I. H., Knottnerus, I. L. H., Staals, J., et al. (2012). Vascular inflammation in cerebral small vessel disease. Neurobiol Aging. 33, 1800-1806. doi: 10.1016/j. neurobiolaging.2011.04.008

Sanders, L. M. J., Hortobágyi, T., Bastide-van Gemert, S. L., van der Zee, E. A., and van Heuvelen, M. J. G. (2019). Dose-response relationship between exercise and cognitive function in older adults with and without cognitive impairment: A systematic review and meta-analysis. PLoS One 14:e0210036. doi: 10.1371/ journal.pone. 0210036

Sangha, P. S., Thakur, M., Akhtar, Z., Ramani, S., and Gyamfi, R. S. (2020). The Link Between Rheumatoid Arthritis and Dementia: A Review. Cureus. 12, e7855. doi: $10.7759 /$ cureus.7855

Sankar, S. B., Pybus, A. F., Liew, A., Sanders, B., Shah, K. J., Wood, L. B., et al. (2019). Low cerebral blood flow is a non-invasive biomarker of neuroinflammation after repetitive mild traumatic brain injury. Neurobiol Dis. 124, 544-554. doi: 10.1016/j.nbd.2018.12.018

Schwartz, D. H., Leonard, G., Perron, M., Richer, L., Syme, C., Veillette, S., et al. (2013). Visceral fat is associated with lower executive functioning in adolescents. Int J Obes (Lond). 37, 1336-1343. doi: 10.1038/ijo.2013.104

Serrano-Pozo, A., and Growdon, J. H. (2019). Is Alzheimer's Disease Risk Modifiable? J Alzheimers Dis. 67, 795-819. doi: 10.3233/JAD181028

Sexton, C. E., Storsve, A. B., Walhovd, K. B., Johansen-Berg, H., and Fjell, A. M. (2014). Poor sleep quality is associated with increased cortical atrophy in community-dwelling adults. Neurology. 83, 967-973. doi: 10.1212/WNL. 000000000000774

Shi, Y., Thrippleton, M. J., Makin, S. D., Marshall, I., Geerlings, M. I., de Craen, A. J. M., et al. (2016). Cerebral blood flow in small vessel disease: A systematic review and meta-analysis. J Cereb Blood Flow Metab. 36, 1653-1667. doi: 10. 1177/0271678X16662891 
Siddarth, P., Burggren, A. C., Eyre, H. A., Small, G. W., and Merrill, D. A. (2018). Sedentary behavior associated with reduced medial temporal lobe thickness in middle-aged and older adults. PLoS One. 13:e0195549. doi: 10.1371/journal. pone.0195549

Singh-Manoux, A., Dugravot, A., Shipley, M., Brunner, E. J., Elbaz, A., Sabia, S., et al. (2018). Obesity trajectories and risk of dementia: 28 years of follow-up in the Whitehall II Study. Alzheimers Dement. 14, 178-186. doi: 10.1016/j.jalz. 2017.06.2637

Slavish, D. C., Graham-Engeland, J. E., Engeland, C. G., Taylor, D. J., and Buxton, O. M. (2018). Insomnia symptoms are associated with elevated C-reactive protein in young adults. Psychol Health. 33, 1396-1415. doi: 10.1080/08870446. 2018.1500577

Smagula, S. F., Stone, K. L., Redline, S., Ancoli-Israel, S., Barrett-Connor, E., Lane, N. E., et al. (2016). Actigraphy- and Polysomnography-Measured Sleep Disturbances, Inflammation, and Mortality Among Older Men. Psychosom Med. 78, 686-696. doi: 10.1097/PSY.0000000000000312

Smith, M. T., Perlis, M. L., Chengazi, V. U., Pennington, J., Soeffing, J., Ryan, J. M., et al. (2002). Neuroimaging of NREM sleep in primary insomnia: a Tc99-HMPAO single photon emission computed tomography study. Sleep 25, 325-335.

Smith, P. J. (2019). Pathways of Prevention: A Scoping Review of Dietary and Exercise Interventions for Neurocognition. Brain Plast. 5, 3-38. doi: 10.3233/ BPL- 190083

Stanek, K. M., Grieve, S. M., Brickman, A. M., Korgaonkar, M. S., Paul, R. H., Cohen, R. A., et al. (2011). Obesity is associated with reduced white matter integrity in otherwise healthy adults. Obesity. 19, 500-504. doi: 10.1038/oby. 2010.312

Steventon, J. J., Foster, C., Furby, H., Helme, D., Wise, R. G., and Murphy, K. (2020). Hippocampal Blood Flow Is Increased After $20 \mathrm{~min}$ of ModerateIntensity Exercise. Cereb Cortex. 30, 525-533. doi: 10.1093/cercor/bhz104

Steyers, C. M. III, and Miller, F. J. Jr. (2014). Endothelial dysfunction in chronic inflammatory diseases. Int J Mol Sci. 15, 11324-11349. doi: 10.3390/ ijms 150711324

Swardfager, W., Yu, D., Ramirez, J., Cogo-Moreira, H., Szilagyi, G., Holmes, M. F., et al. (2017). Peripheral inflammatory markers indicate microstructural damage within periventricular white matter hyperintensities in Alzheimer's disease: a preliminary report. Alzheimers Dement. 7, 56-60. doi: 10.1016/j.dadm.2016.12.011

Tabuchi, S. (2014). Auditory dysfunction in patients with cerebrovascular disease. Sci World J. 4, 261824. doi: 10.1155/2014/261824

ter Telgte, A., van Leijsen, E. M. C., Wiegertjes, K., Klijn, C. J. M., Tuladhar, A. M., and de Leeuw, F. E. (2018). Cerebral small vessel disease: from a focal to a global perspective. Nat Rev Neurol. 14, 387-398. doi: 10.1038/s41582-018-0014-y

Tsai, C. L., Ukropec, J., Ukropcová, B., and Pai, M. C. (2017). An acute bout of aerobic or strength exercise specifically modifies circulating exerkine levels and neurocognitive functions in elderly individuals with mild cognitive impairment. Neuroimage Clin. 17, 272-284. doi: 10.1016/j.nicl.2017.10.028

Uzoni, A., Ciobanu, O., Sandu, R. E., Buga, A. M., and Popa-Wagner, A. (2015). Life style, Perfusion deficits and Co-morbidities Precipitate Inflammation and Cerebrovascular Disorders in Aged Subjects. Discoveries (Craiova) 3, e39. doi: 10.15190/d.2015.31

Van der Veen, P. H., Muller, M., Vincken, K. L., Hendrikse, J., Mali, W. P., van der Graaf, Y., et al. (2015). Longitudinal relationship between cerebral small-vessel disease and cerebral blood flow: the second manifestations of arterial diseasemagnetic resonance study. Stroke. 46, 1233-1238. doi: 10.1161/STROKEAHA. 114.008030

Verhaaren, B. F. J., Debette, S., Bis, J. C., Smith, J. A., Ikram, M. K., Adams, H. H., et al. (2015). Multiethnic genome-wide association study of cerebral white matter hyperintensities on MRI. Circ Cardiovasc Genet. 8, 398-409. doi: 10.1161/CIRCGENETICS.114.000858

Vermeer, S. E., Longstreth, W. T., and Koudstaal, P. J. (2007). Silent brain infarcts: a systematic review. Lancet Neurol. 6, 611-619. doi: 10.1016/S1474-4422(07) 70170-9

Walker, K. A., Power, M. C., Hoogeyeen, R. C., Folsom, A. R., Ballantyne, C. M., Knopman, D. S., et al. (2017). Midlife Systemic Inflammation, Late- Life White
Matter Integrity, and Cerebral Small Vessel Disease: The Atherosclerosis Risk in Communities Study. Stroke 48, 3196-3202. doi: 10.1161/strokeaha.117.018675

Wardlaw, J. M., Valdés Hernández, M. C., and Muñoz-Maniega, S. (2015). What are white matter hyperintensities made of? Relevance to vascular cognitive impairment. J Am Heart Assoc. 4, 001140. doi: 10.1161/JAHA.114.00 1140

Whitmer, R. A., Gustafson, D. R., Barrett-Connor, E., Haan, M. N., Gunderson, E. P., and Yaffe, K. (2008). Central obesity and increased risk of dementia more than three decades later. Neurology 71, 1057-1064. doi: 10.1212/01.wnl. 0000306313.89165.ef

Willeumier, K. C., Taylor, D. V., and Amen, D. G. (2011). Elevated BMI Is Associated With Decreased Blood Flow in the Prefrontal Cortex Using SPECT Imaging in Healthy Adults. Obesity. 19, 1095-1097. doi: 10.1038/oby. 2011.16

Windsor, A. M., and Ruckenstein, M. J. (2018). “Anti-inflammatory Therapies for Sensorineural Hearing Loss," in Inflammatory Mechanisms in Mediating Hearing Loss, eds V. Ramkumar and L. Rybak (Cham: Springer), doi: 10.1007/ 978-3-319-92507-3_10

World Health Organization. (2017). Global action plan on the public health response to dementia 2017-2025. Geneva: World Health Organization.

Xie, L., Kang, H., Xu, Q., Chen, M. J., Liao, Y., Thiyagarajan, M., et al. (2013). Sleep drives metabolite clearance from the adult brain. Science. 342, 373-377. doi: $10.1126 /$ science. 1241224

Xu, W. L., Atti, A. R., Gatz, M., Pedersen, N. L., Johansson, B., and Fratiglioni, L. (2011). Midlife overweight and obesity increase late-life dementia risk: a population-based twin study. Neurology. 76, 1568-1574. doi: 10.1212/WNL. 0b013e3182190d09

Yamashiro, K., Tanaka, R., Tanaka, Y., Miyamoto, N., Shimada, Y., Ueno, Y., et al. (2014). Visceral fat accumulation is associated with cerebral small vessel disease. Eur J Neurol. 21, 667-673. doi: 10.1111/ene.12374

Yang, G., Lai, C. S., Cichon, J., Ma, L., Li, W., and Gan, W. B. (2014). Sleep promotes branch-specific formation of dendritic spines after learning. Science. 344, 1173-1178. doi: 10.1126/science.1249098

Yiannopoulou, K. G., Anastasiou, A. I., Zachariou, V., and Pelidou, S. H. (2019). Reasons for Failed Trials of Disease-Modifying Treatments for Alzheimer Disease and Their Contribution in Recent Research. Biomedicines. 7, 97. doi: 10.3390/biomedicines7040097

Zahorska-Markiewicz, B., Janowska, J., Olszanecka-Glinianowicz, M., and Zurakowski, A. (2000). Serum concentrations of tnf-alpha and soluble tnfalpha receptors in obesity. Int J Obes Relat Metab Disord. 24, 1392-1395. doi: 10.1038/sj.ijo.0801398

Zhang, B., Wang, H. E., Bai, Y. M., Tsai, S. J., Su, T. P., Chen, T. J., et al. (2020). Inflammatory bowel disease is associated with higher dementia risk: a nationwide longitudinal study. Gut 70, 85-91. doi: 10.1136/gutjnl-2020320789

Zhou, F., Huang, M., Gu, L., Hong, S., Jiang, J., Zeng, X., et al. (2019). Regional cerebral hypoperfusion after acute sleep deprivation: A STROBE-compliant study of arterial spin labeling fMRI. Medicine (Baltimore). 98, e14008. doi: 10.1097/MD.0000000000014008

Zlatar, Z. Z., Hays, C. C., Mestre, Z., Campbell, L. M., Meloy, M. J., Bangen, K. J., et al. (2019). Dose-dependent association of accelerometer-measured physical activity and sedentary time with brain perfusion in aging. Exp Gerontol. 125, 110679. doi: 10.1016/j.exger.2019.110679

Conflict of Interest: The author declares that the research was conducted in the absence of any commercial or financial relationships that could be construed as a potential conflict of interest.

Copyright (C) 2021 Hakim. This is an open-access article distributed under the terms of the Creative Commons Attribution License (CC BY). The use, distribution or reproduction in other forums is permitted, provided the original author(s) and the copyright owner(s) are credited and that the original publication in this journal is cited, in accordance with accepted academic practice. No use, distribution or reproduction is permitted which does not comply with these terms. 